\title{
TaMYB3, Encoding a Functional MYB Transcriptor, Isolated from the Purple Pericarp of Triticum aestivum
}

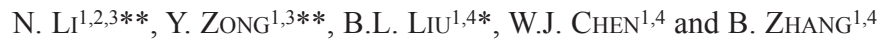 \\ ${ }^{1}$ Key Laboratory of Adaptation and Evolution of Plateau Biota (AEPB), \\ Northwest Institute of Plateau Biology, Chinese Academy of Sciences, Qinghai Xining 810008, China \\ ${ }^{2}$ Qinghai University, Qinghai Xining 810014, China \\ ${ }^{3}$ University of Chinese Academy of Sciences, Beijing 100049, China \\ ${ }^{4}$ Qinghai Province Key Laboratory of Crop Molecular Breeding, Xining 810008, China
}

(Received 25 September 2016; Accepted 17 January 2017;

Communicated by H. Grausgruber)

\begin{abstract}
Purple pericarp is an interesting and useful trait in Triticum aestivum, but the molecular mechanism behind this phenotype remains unclear. The allelic variation in the MYB transcriptors is associated with the phenotype of pigmented organs in many plants. In this study, a $M Y B$ transcription factor gene, TaMYB3, was isolated using homology-based cloning and a differentially expressed gene mining approach, to verify the function of the MYB transcriptor in the purple pericarp. The coding sequence of TaMYB3 in cultivar Gy115 was the same as that in cultivar Opata. TaMYB3 was localized to FL0.62-0.95 on chromosome 4BL. The TaMYB3 protein contains DNA-binding and transcription-activation domains, and clustered on a phylogenetic tree with the MYB proteins that regulates anthocyanin and proanthocyanin biosynthesis. TaMYB3 localized in the nuclei of Arabidopsis thaliana and wheat protoplasts after it was transiently expressed with PEG transformation. TaMYB3 induced anthocyanin synthesis in the pericarp cells of Opata in the dark in collaboration with the basic helix-loop-helix protein $\mathrm{ZmR}$, which is also the function of $\mathrm{ZmC1}$. However, TaMYB3 alone did not induce anthocyanin biosynthesis in the pericarp cells of the white grain wheat cultivar Opata in the light after bombardment, whereas the single protein ZmR did. Light increased the expression of TaMYB3 in the pericarp of Gy115 and Opata, but only induced anthocyanin biosynthesis in the grains of Gy115. Our results extend our understanding of the molecular mechanism of the purple pericarp trait in T. aestivum.
\end{abstract}

Keywords: MYB transcriptor, purple pericarp, anthocyanin biosynthesis, Triticum aestivum

\section{Introduction}

Anthocyanin is a water-soluble pigment that occurs ubiquitously in terrestrial plants, except cacti and beets. This class of compound endows plant organs with a red, pink, blue, or purple color. Anthocyanin also has health-giving properties, including antioxidant, antiviral, and antitumor activities (Baublis et al. 2000). Because it is rich in anthocyanins, purple-grained wheat is increasingly regarded as a health food.

\footnotetext{
*Corresponding author; E-mail: blliu@nwipb.cas.cn

**These authors contributed to this manuscript equally
} 
Purple-grained wheat can also be used as an effective marker of the natural outcrossing rate (Griffin 1987). The purple pericarp trait in common wheat was once believed to have originated from the tetraploid wheat of Ethiopia, and is dominant over white grain and red grain (Zeven 1991). Two complementary genes, Pp1 and Pp3, located on chromosomes $7 \mathrm{~B}$ and 2A, respectively, control the purple grain trait (Khlestkina et al. 2010). There are also genes homologous to Ppl on chromosomes 7A and 7D (Tereshchenko et al. 2012; Gordeeva et al. 2015). The purple grain trait in common wheat has been widely studied with classical genetics, but its molecular mechanism has not been fully explained, because the genome of common wheat is large, with three homologous chromosomes.

The key genes in the anthocyanin biosynthesis pathway have been isolated by mutant analyses and map-based cloning in Arabidopsis, maize, tobacco, and other model plants with small genomes, because the mutants are readily observable. The main structural genes of anthocyanin biosynthesis include phenylalanine ammonia lyase, chalcone synthase, chalcone isomerase, flavanone 3-hydroxylase, flavonoid 3-hydroxylase, dihydroflavonol 4-reductase, leucoanthocyanidin dioxygenase, and flavonoid 3-O-glucosyltransferase (Dunlop and Curtis 1991). The metabolism and regulation of anthocyanin biosynthesis are mainly achieved through the regulated expression of structural genes (Gion et al. 2012; Xu et al. 2015), and three types of transcription factors, MYB, bHLH, and WD40, regulate the expression of these structural genes (Broun 2005; Koes et al. 2005). The allelic variation in the $M Y B$ genes plays a key role in the color differentiation of natural plant populations. For instance, nonfunctional $V v M Y B A 1$ is present in the white grape cultivar Pinot Noir, and the restoration of $V v M Y B A 1$ causes the red skin of the grape cultivar Pinot Blanc as a result of intra-long-terminal-repeat (intra-LTR) recombination within a retrotransposon in the gene's promoter (Kobayashi et al. 2005). Similar functional MYB regulators also exist in Arabidopsis (AtMYB75 [PAP1]) and AtMYB90 [PAP2]) (Borevitz et al. 2000), petunia (AN2) (Quattrocchio et al. 1999), sweet potato (MYB1) (Mano et al. 2007), legumes (LAP1) (Peel et al. 2009), and Epimedium sagittatum (EsMYBA1) (Huang et al. 2013). Among the genes associated with anthocyanin biosynthesis in Triticum aestivum, the red grain $(R)$ and red coleoptile $(R c)$ genes have been shown to encode R2R3-MYB transcription factors that regulate proanthocyanidin and anthocyanin biosynthesis in grains and coleoptiles, respectively (Himi et al. 2011; Himi and Taketa 2015; Wang et al. 2016). The candidate gene for the purple pericarp trait in common wheat, Pp3, is the basic helix-loop-helix (bHLH) transcriptor TaMYC1 (Shoeva et al. 2014; Liu et al. 2016), and the $P p-1$ genes are thought to be orthologues of maize $C 1$ and rice $\mathrm{OsCl}$, which encode MYB-like transcription factors responsible for the activation of the structural genes encoding various enzymes that participate in anthocyanin synthesis (Saitoh et al. 2004; Khlestkina 2013). However, the function of the MYB transcriptor in the purple grain trait of common wheat has not been analyzed.

In this study, a MYB transcription factor gene, TaMYB3, was isolated from purple grains with a homology cloning technology based on $\mathrm{ZmCl}$ and $\mathrm{OsCl}$. The chromosomal site, subcellular localization, functional verification, and expression profile of the gene were analyzed to evaluate the role of TaMYB3 in the anthocyanin biosynthesis underlying the purple grain trait. 


\section{Materials and Methods}

\section{Plant materials}

Wheat cultivar Gy115, with the purple grain trait, was bred at the Northwest Institute of Plateau Biology, Chinese Academy of Sciences. The white grain wheat cultivars Opata, Aegilops tauschii AS69 (Accession number: PI603252), and T. urartu IE29-1 (Accession number: PI428183), the chromosomal substitution lines of Chinese Spring (CS) in durum wheat Langdon (Joppa and Williams 1988) and the chromosomal deletion lines of CS (Endo and Gill 1996) were provided by the National Plant Germplasm System (NPGS) of the United States Department of Agriculture (http://www.ars-grin.gov/).

\section{DNA and RNA extraction}

All samples were collected in liquid nitrogen, and stored in $-80{ }^{\circ} \mathrm{C}$ before DNA and RNA extraction. DNA was extracted using the CTAB method, and total RNA was extracted from the samples with TRIzol Reagent (Invitrogen, Burlington, ON, Canada), according to the manufacturer's protocol. The DNA and RNA contents were measured with the BioPhotometer Plus (Eppendorf), and $2 \mu \mathrm{g}$ of RNA was used for the reverse transcription reactions.

\section{PCR and semi-quantitative PCR}

PCR amplification was performed with Phusion High-Fidelity DNA Polymerase (Thermo-Fisher Scientific, Shanghai, China) in the GeneAmp PCR System 9700 (ThermoFisher Scientific), with the following thermal cycling parameters: 2 min of denaturation at $98{ }^{\circ} \mathrm{C}$; 35 cycles of $15 \mathrm{~s}$ at $98^{\circ} \mathrm{C}, 30 \mathrm{~s}$ at $61^{\circ} \mathrm{C}$, and $30 \mathrm{~s}$ at $72{ }^{\circ} \mathrm{C}$; followed by a final extension of $5 \mathrm{~min}$ at $72{ }^{\circ} \mathrm{C}$. The PCR products were extracted from $1.0 \%$ agarose gels using the Tiangen TIANgel Midi Purification Kit (Tiangen) and cloned into the pGEM-T Easy vector (Promega Corporation, Madison, Wisconsin, USA). Escherichia coli DH5 $\alpha$ cells were then transformed with the recombinant plasmid, and six positive clones were sent to a commercial company (Huada Gene, Shenzheng, China) for sequencing. The nucleotide sequence of TaMYB3 was deposited in GenBank under accession number KX373488. All the primers used in this study are listed in Table S1*. In all the semiquantitative RT-PCR experiments conducted in this study, the amplification of the wheat tubulin gene transcripts was used to normalize the cDNA contents of the various reverse transcription mixtures. Thirty-two thermocycles were used to check the linearity of the amplifications. The reproducibility of the transcription patterns determined with semiquantitative PCR was tested in at least three independent assays.

*Further details about the Electronic Supplementary Material (ESM) can be found at the end of the article. 


\section{Bioinformatic analysis}

The sequences were aligned with the Vector NTI 10 software (Thermo-Fisher Scientific). Primers were designed with the Primer5 software (Premier Biosoft, Palo Alto, CA, USA). Phylogenetic trees were constructed with the MEGA 4 software (Tamura et al. 2007), and the protein domains were predicted with the web software SMART (http://smart.em BLheidelberg.de/).

Transient expression in protoplasts transformed with the polyethylene glycol (PEG) method

For the subcellular localization experiment, the coding sequence of TaMYB3 without the termination codon was ligated into the PMNG vector after the ubiquitin promoter and before the enhanced green fluorescent protein gene $(e G F P)$. Protoplasts were prepared according to Sheen (2002) and Yoo et al. (2007). The leaves of Arabidopsis (without blossom) were used to prepare the protoplasts, and etiolated wheat seedlings 7 days after germination were used to prepare the wheat protoplasts. The protoplasts were transformed with plasmid PMNG:TaMYB3 or PMNG (the positive control) using a PEG method, based on the method of Sheen (2002).

\section{Expression of TaMYB3 in grains after dark or light treatment}

To study the reaction of TaMYB3 and anthocyanin biosynthesis to light, two rows of spike glumes were removed from Gy115 14 days after blossoming as the light treatment, and the remaining two rows were retained as the dark treatment. During the daytime, the spike glumes can reduce the light intensity, so grains without glumes must be exposed to direct sunlight. The grains subjected to the dark and light treatments were stored at $-80{ }^{\circ} \mathrm{C}$ before photography and the gene expression analysis. The photographs were taken with an Canon D500 SLR camera.

\section{Transient expression in pericarps}

The coding sequences of the TaMYB3, $\mathrm{ZmCl}$, and $\mathrm{ZmR}$ genes from maize were cut with restriction enzymes $B a m H I$ and $K p n I$ and ligated into the PUBI vector after the Ubi promoter. To transform the cells, the plasmids were shot into them with a gene gun, according to the method of Ahmed, for functional verification (Ahmed et al. 2003). The grains were selected for bombardment 14 days after blossoming. After bombardment, the Petri dishes containing the grains of Gy115 were wrapped in aluminum for the dark treatment, and the Petri dishes of Opata were placed directly in a light incubator for the light treatment (light intensity $100 \mu \mathrm{mol} / \mathrm{s} / \mathrm{m}^{2}, 23{ }^{\circ} \mathrm{C}, 16 / 8 \mathrm{~h} \mathrm{light} /$ dark). The grains were observed and photographed after 2 days under a stereomicroscope (Leica Co.). 


\section{Results}

\section{Molecular characteristics of TaMYB3}

After the sequences of $\mathrm{ZmCl}$ of maize, AtTT2 of $A$. thaliana, and $\mathrm{OsCl}$ of rice were aligned, the conserved primers $\mathrm{TaC} 1-\mathrm{F}$ and $\mathrm{TaC} 1-\mathrm{R}$ spanning two introns were designed to isolate the $M Y B$ genes regulating anthocyanin biosynthesis in pigmented grains using homology-based cloning (Fig. S1A). Previous studies have demonstrated that anthocyanin biosynthesis is induced by light in the purple pericarp tissue of wheat (Wang et al. 2011). The grains of Gy 115 treated with light or dark were used as the substrates in which to distinguish the differential expression of the $M Y B$ gene. A cDNA amplification product was isolated from 7-day grain from wheat cultivar Gy115 treated with light for 2 days, but was not isolated from grains exposed to the dark treatment (Fig. S1B). The nucleotide sequence of amplified fragment was very similar to that of the MYB genes in the alignment. Contigs TA59663_4565 and TA59683_4565 were recovered from the expressed sequence tag (EST) database for wheat at the TIGR website with a BLAST search. The two contigs were assembled into one sequence of $1231 \mathrm{bp}$. TaMYB3 was isolated from Gy115 and Opata based on the assembled sequence. The genomic sequence of TaMYB3 in Opata differed by a single base in the intron from that in Gy115, and did not influence the amino acid sequence of the encoded protein.

On a phylogenetic tree, TaMYB3, ZmC1, ZmP, and AtTT2 clustered on the same branch (Fig. 1B). With the exception of TaMYB3, all these genes have been shown to encode proteins that regulate anthocyanin or proanthocyanin biosynthesis. $\mathrm{ZmC1}$ and TaMYB3 share 54\% sequence identity, and in previous research, $\mathrm{ZmC1}$ has been shown to have special DNA-binding and transcription-activation domains that promote the expression of the structural genes involved in anthocyanin biosynthesis (Grotewold et al. 2000). Based on these findings, the amino acid sequence of TaMYB3 was compared with that of $\mathrm{ZmC1}$. Although TaMYB3 is 29 amino acids shorter than ZmC1, TaMYB3 has an integrated DNA-binding domain of 101 amino acids, which is similar to that of $\mathrm{ZmC1}$, with only 13 single-amino-acid substitutions, and a transcription domain of 39 amino acids with a 7-amino-acid deletion and 12 single-amino-acid substitutions (Fig. 1A). Interestingly, TaMYB3 has only one intron, whereas the other $M Y B$ genes have two introns in their gene structures (Fig. 1C).

\section{Chromosomal position and subcellular localization}

IE29-1 (T. urartu) and AS69 (Ae. tauschii), the Langdon (CS) chromosome substitution lines and the CS chromosome deletion lines were used to identify the position of TaMYB3 on the wheat chromosomes. TaMYB3 could not be amplified from the genome of IE29-1 (genome donor A) or AS69 (genome donor D), signifying that TaMYB3 does not occur in genome A or D (Fig. 2A). However, TaMYB3 was detected in the Langdon genome, whereas it was not amplified from the substitution line lacking the $4 \mathrm{~B}$ chromosome of Langdon but containing the 4D chromosome of CS (Fig. 2A). Therefore, TaMYB3 must occur on chromosome 4B. To confirm the position of TaMBY3 on chromosome 4B, a se- 


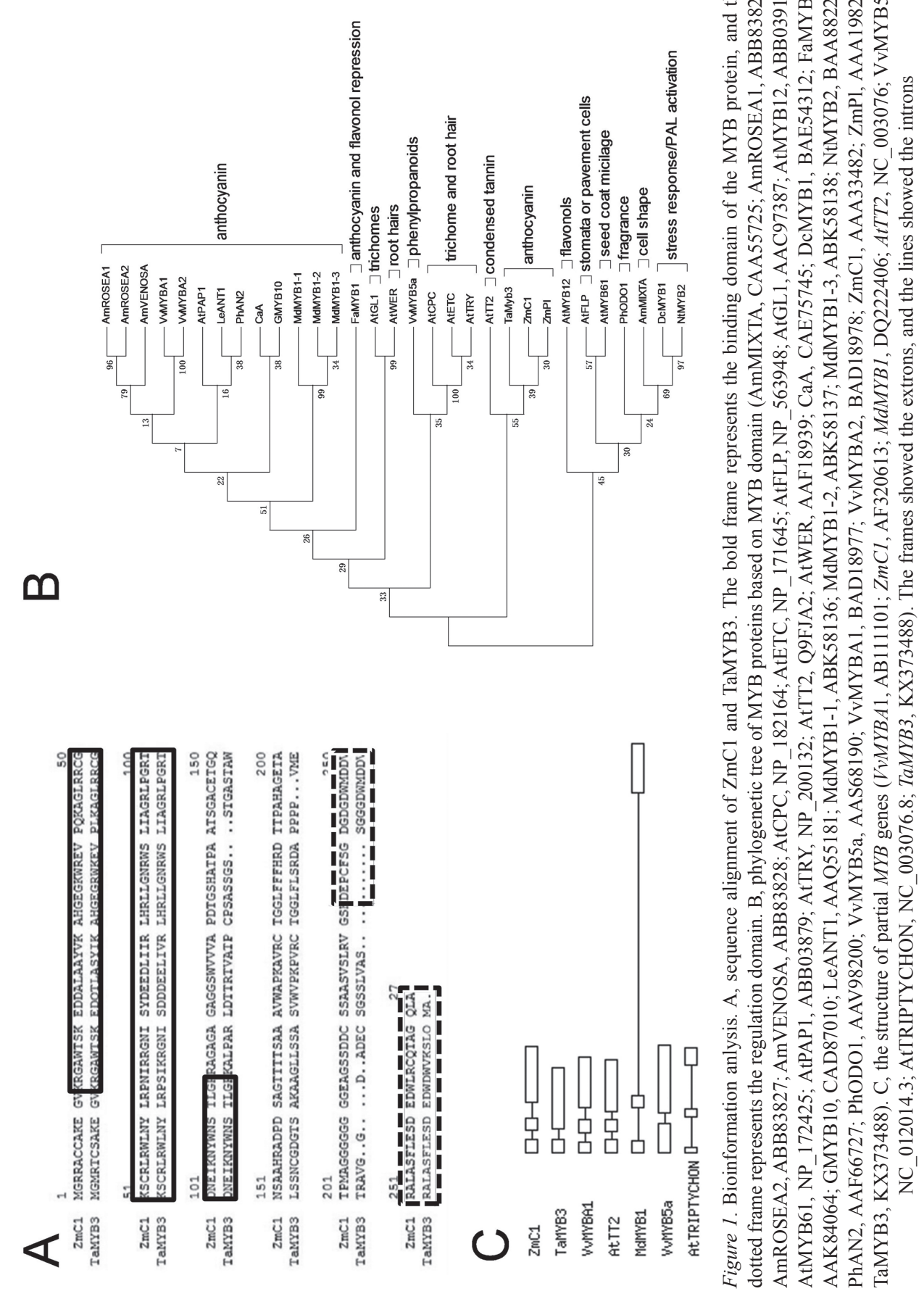

Cereal Research Communications 45, 2017 
A

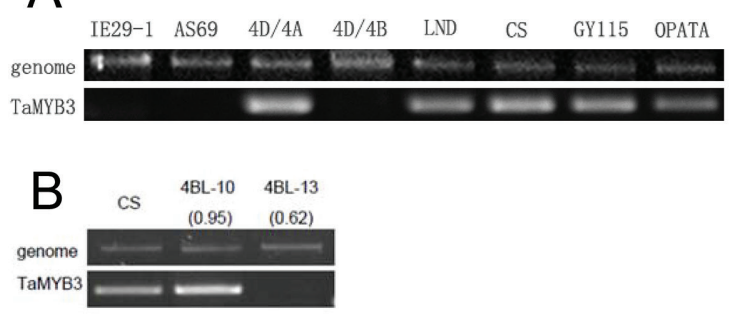

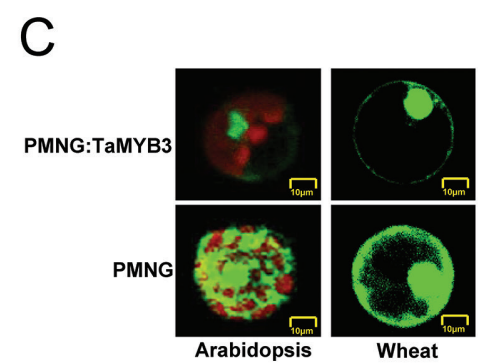

Arabidopsis

Wheat

Figure 2. Chromosome residential site and subcellular location of TaMYB3. (A) IE29-1, Einkorn wheat (genome A); AS69, Ae. tauschii (genome D); 4D/4A, Langdon 4A (CS 4D); 4D/4B, Langdon 4B (CS 4D); LND, Langdon, Triticum durum (genome AB); CS, Chinese Spring, common wheat (genome ABD); Gy115, common wheat, Opata, common wheat. Genome land was $100 \mathrm{ng}$ genome DNA, and TaMYB3 land was amplification products with TaMYB3 primer. (B) CS, Chinese Spring. 4BL10, CS with $95 \%$ of long arm of chromosome 4B; 4BL13, CS with $62 \%$ of long arm of chromosome 4B. Genome land was 100 ng genome DNA, and TaMYB3 land was amplification products with TaMYB3 primer. (C) Protoplasts under confocal laser scanning microscopy after transient expression with plasmids

ries of CS 4B deletion lines was used. TaMBY3 was detected at 4BL10 (FL0.95), but not at 4BL13 (FL0.62) (Fig. 2B), so TaMYB3 should occur at FL0.62-0.95 on the long arm of chromosome 4B (Fig. S2). When Arabidopsis and wheat protoplasts were transformed with PMNG, eGFP was ubiquitously expressed in the protoplasts, whereas the fusion protein TaMYB3-eGFP was only present in the nuclei of the protoplasts (Fig. 2C). Therefore, we infer that TaMYB3 is located in the nuclei.

\section{Expression profile of TaMYB3 in grains treated with light or dark}

The light treatment for two days induced anthocyanin biosynthesis in the grains of Gy115, but not in those of Opata. The expression of TaMYB3 differed from the expression of anthocyanin biosynthesis, and was greater after the light treatment than after the dark treatments in both Gy115 and Opata (Fig. 3). This phenotype may be attributable to the

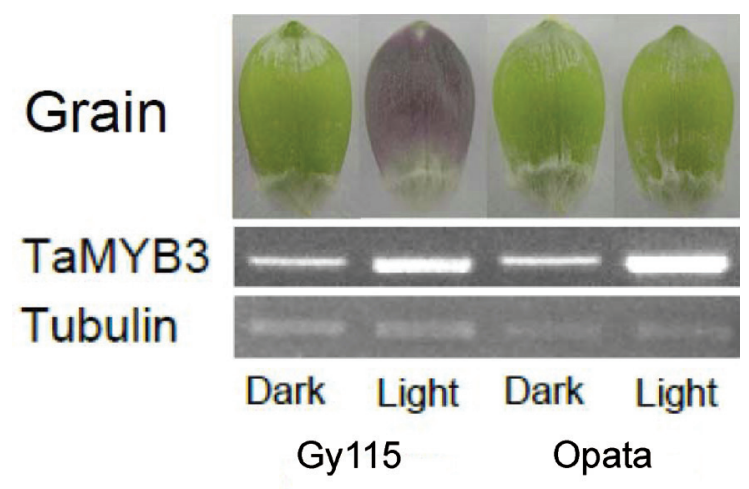

Figure 3. Relative transcript levels of TaMYB3 and Tubulin in grains with dark treatment and light treatment 

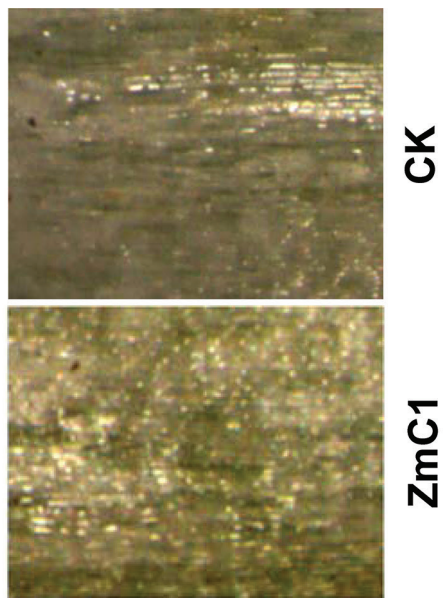

究

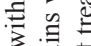

उ

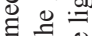

要

¿ $₫$

تृं

की

要离

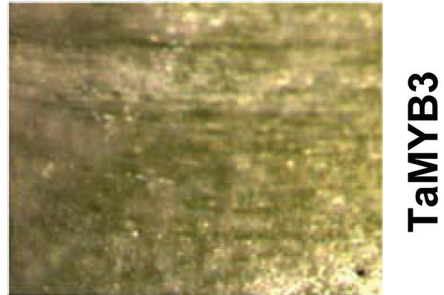

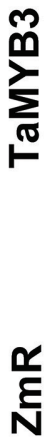

焉苛

㻤

$\vec{g}$

क त त

乙艹

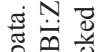

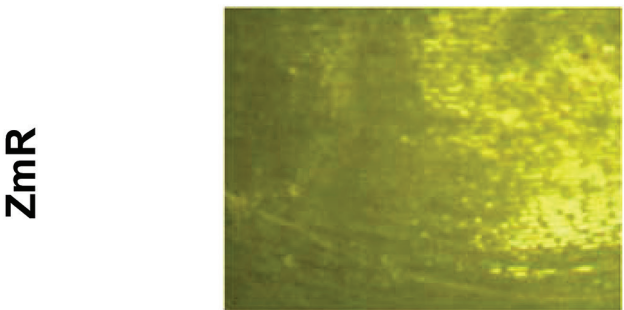

에용
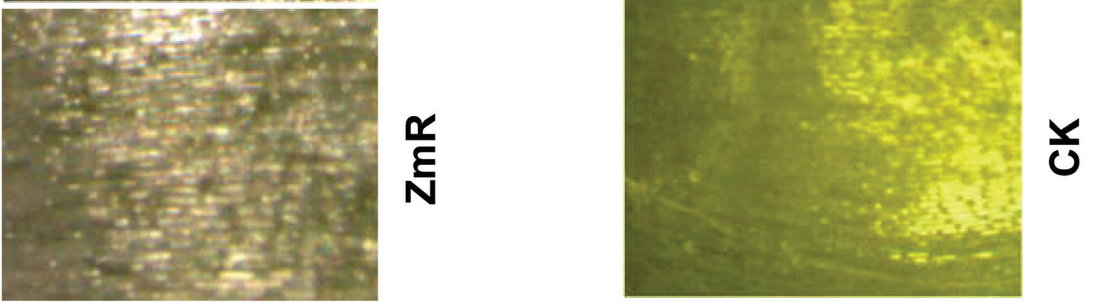

की व्यें

율

$\mathbf{U}$

政

氞苛

코

菏

클

है

है है
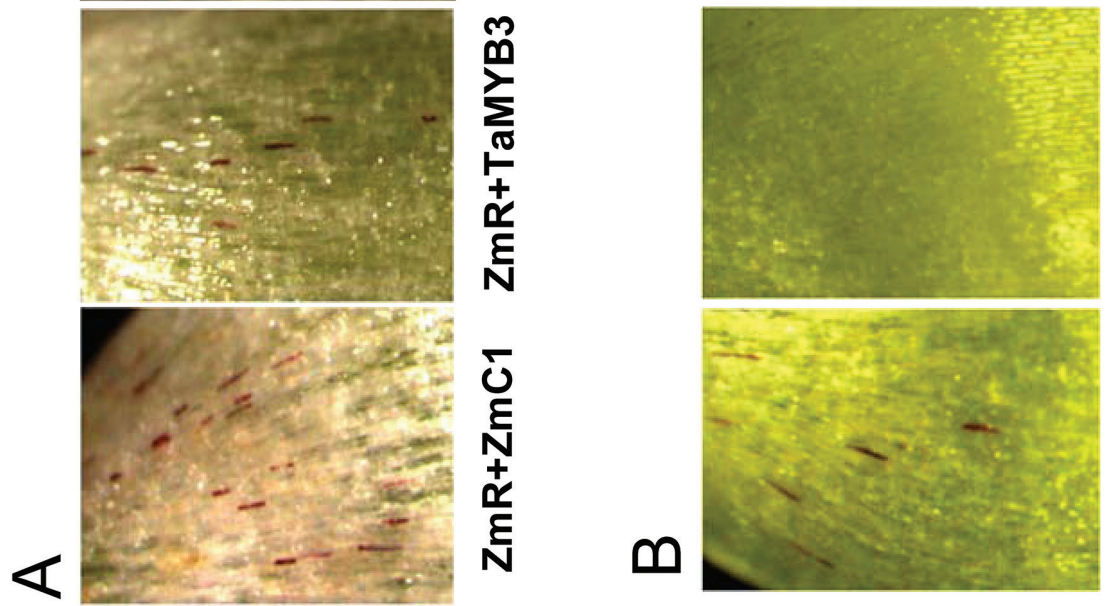

$m$ 节 导

m

๖

$\sum_{0}$

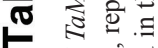
पै

.

के के

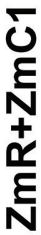
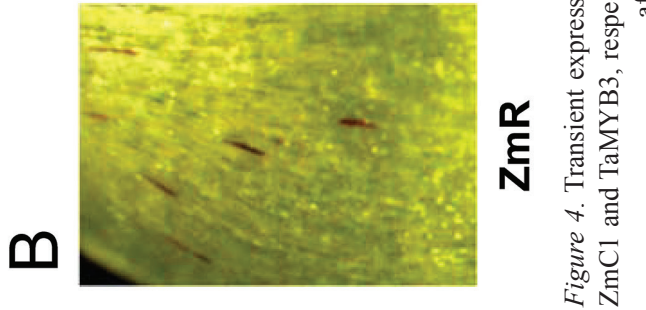

Cereal Research Communications 45, 2017 
fact that light induced the expression of TaMYB3 and anthocyanin biosynthesis in Gy115, and that anthocyanin provided the pericarp with some protection from light. Light induced the expression of TaMYB3, but did not cause anthocyanin biosynthesis without the help of other genes related to anthocyanin biosynthesis in the grains of Opata.

\section{Transient expression of TaMYB3 in the pericarp}

TaMYB3 was very similar to $Z m C 1$ in the evolutionary analysis, and in previous research, $\mathrm{ZmC1}$ was shown to induce anthocyanin biosynthesis when coexpressed with the $b H L H$ gene (Cone et al. 1986; Ahmed et al. 2003). In the present study, the bHLH regulators $\mathrm{ZmR}$ and $\mathrm{ZmCl}$ were isolated from maize to compare the functions of TaMYB3 and $\mathrm{ZmC1}$. The transient expressions of TaMYB3 or $\mathrm{ZmCl}$ induced anthocyanin biosynthesis in the pericarp cells of GY115 after dark treatment, with the assistance of $Z m R$. However, the expression of TaMYB3, $\mathrm{ZmC1}$, or $\mathrm{ZmR}$ alone did not produce red cells in the pericarps (Fig. 4A). Therefore, TaMYB3 functions in regulating anthocyanin biosynthesis in a similar to $\mathrm{ZmC1}$. TaMYB3, ZmC1, or ZmR was also transiently expressed in the white pericarps of Opata in the light. ZmR alone induced anthocyanin biosynthesis in the pericarp cells of Opata with light treatment, whereas TaMYB3 alone did not produce the same phenotype (Fig. 4B).

\section{Discussion}

In this study, we hoped to identify the gene encoding the key factor that activates anthocyanin biosynthesis in the wheat pericarp, as in our recent study (Liu et al. 2016). Compared with the transcriptomic analysis used in the previous study, we used homologybased cloning and differentially expressed gene mining here. The MYB transcriptors $\mathrm{ZmC1}$, AtTT2, and OsC1 have been shown to regulate anthocyanin biosynthesis in maize, Arabidopsis, and rice, respectively (Cone et al. 1986; Saitoh et al. 2004; Wei et al. 2007). The MYB transcriptor sequences conserved in maize, $A$. thaliana, and rice should also be conserved in common wheat. TaMYB3, the protein derived from the conserved nucleotide sequence detected, has DNA-binding and regulatory domains involved in anthocyanin biosynthesis. On a phylogenetic tree, TaMYB3 clustered on the same branch as $\mathrm{ZmC1}$ and other proteins that regulate anthocyanin and proanthocyanin biosynthesis. The sequence-conservation-based strategy used to isolate the homologous gene was very efficient. The transient expression of TaMYB3 induced anthocyanin biosynthesis in the pericarp of cultivar Gy115 during dark treatment, with the co-operation of the bHLH transcription factor $Z m R$, which is similar to $Z m C 1$ (Ahmed et al. 2003). TaMYB3 is expressed in purple grains and is induced by light. Following the increased expression of TaMYB3, anthocyanin began to be synthesized. Therefore, TaMYB3 regulates anthocyanin biosynthesis and could be responsible for the purple pericarp, indicating that TaMYB3 is involved in anthocyanin biosynthesis in the purple pericarp, even if other MYB transcriptors also participate in the process. 
According to our experiments, the TaMYB3 gene occurs at FL0.64-0.95 on wheat chromosome 4BL, unlike $R, R c$, and $P p$. $R$ and $R c$ occur on chromosomes 3 and 7, respectively (Himi et al. 2011; Himi and Taketa 2015; Wang et al. 2016), and $P p 1$ and $P p 3$, which control the purple grain trait, are located on chromosomes $7 \mathrm{~B}$ and $2 \mathrm{~A}$, respectively (Khlestkina et al. 2010). Therefore, the chromosomal site of TaMYB3 is clearly inconsistent with the chromosomal locations of the two complementary genes responsible for the purple grain trait of wheat. The genomic and cDNA sequences of TaMYB3 in Opata are the same as those in Gy115, except for one single-nucleotide difference in the intron. Its relative expression profile in the grains of Opata is also similar to that in the grains of Gy115. Therefore, TaMYB3 is not the key gene responsible for the purple pericarp of common wheat. However, its chromosomal location at FL0.64-0.95 is close to another locus on 4BL that determines the purple coleoptile trait (Khlestkina et al. 2002). The transient expression of TaMYB3 alone did not induce anthocyanin biosynthesis in the white pericarp of Opata, whereas the transient expression of $\mathrm{ZmR}$ did. As mentioned in the introduction, the bHLH transcriptor TaMYC1 was thought to be encoded by the candidate gene for Pp3 (Shoeva et al. 2014; Liu et al. 2016). The transient expression of $Z m R$ compensated for TaMYC1 in the white pericarp of Opata. This also suggests that the native Opata TaMYB3 protein interacted with the transiently expressed $\mathrm{ZmR}$, resulting in anthocyanin synthesis under light condition. $P p 3$ requires the help of $P p 1$ to exercise its function in inducing anthocyanin biosynthesis in the pericarp of common wheat (Khlestkina et al. 2010). More work is required to identify the gene encoding Pp1.

In this study, a typical MYB transcriptor gene, TaMYB3, was isolated from the purple pericarp of common wheat. TaMYB3 regulates anthocyanin biosynthesis, and is probably involved in the anthocyanin biosynthesis that causes the purple pericarp. However, $\mathrm{Ta}$ $M Y B 3$ is not the key gene responsible for the purple pericarp because its chromosomal location is inconsistent with this role and there is no obvious difference in its expression in the purple cultivar Gy115 and the white cultivar Opata. However, these results should facilitate the further exploration of the molecular mechanism underlying the purple pericarp trait.

\section{Acknowledgements}

This research was financially supported by the West Light Foundation of the Chinese Academy of Sciences, the Basic Research Projects of Qinghai Province (2015-ZJ-701), and the Pilot Projects of Designer Breeding by Molecular Modules, Chinese Academy of Sciences.

\section{References}

Ahmed, N., Maekawa, M., Utsugi, S., Himi, E., Ablet, H., Rikiishi, K., Noda, K. 2003. Transient expression of anthocyanin in developing wheat coleoptile by maize $C 1$ and B-peru regulatory genes for anthocyanin synthesis. Breed. Sci. 53:29-34.

Baublis, A.J., Lu, C.R., Clydesdale, F.M., Decker, E.A. 2000. Potential of wheat-based breakfast cereals as a source of dietary antioxidants. J. Am. Coll. Nutr. 19:308-311. 
Borevitz, J.O., Xia, Y., Blount, J., Dixon, R.A., Lamb, C. 2000. Activation tagging identifies a conserved MYB regulator of phenylpropanoid biosynthesis. Plant Cell 12:2383-2394.

Broun, P. 2005. Transcriptional control of flavonoid biosynthesis: a complex network of conserved regulators involved in multiple aspects of differentiation in Arabidopsis. Curr. Opin. Plant Biol. 8:272-279.

Cone, K.C., Burr, F.A., Burr, B. 1986. Molecular analysis of the maize anthocyanin regulatory locus $C 1$. Proc. Natl Acad. Sci. USA 83:9631-9635.

Dunlop, D.S., Curtis, W.R. 1991. Synergistic response of plant hairy-root cultures to phosphate limitation and fungal elicitation. Biotechnol. Prog. 7:434-438.

Endo, T.R., Gill, B.S. 1996. The deletion stocks of common wheat. J. Hered. 87:295-307.

Gion, K., Suzuri, R., Ishiguro, K., Katsumoto, Y., Tsuda, S., Tanaka, Y., Mouradova, E., Brugliera, F., Chandler, S. 2012. Genetic engineering of floricultural crops: Modification of flower colour, flowering and shape. Acta Hort. 953:209-216.

Gordeeva, E.I., Shoeva, O.Y., Khlestkina, E.K. 2015. Marker-assisted development of bread wheat near-isogenic lines carrying various combinations of purple pericarp $(P p)$ alleles. Euphytica 203:469-476.

Griffin, W.B. 1987. Outcrossing in New Zealand wheats measured by occurrence of purple grain. New Zealand J. Agric. Res. 30:287-290.

Grotewold, E., Sainz, M.B., Tagliani, L., Hernandez, J.M., Bowen, B., Chandler, V.L. 2000. Identification of the residues in the Myb domain of maize $\mathrm{C} 1$ that specify the interaction with the bHLH cofactor R. Proc. Natl. Acad. Sci. USA 97:13579-13584.

Himi, E., Maekawa, M., Miura, H., Noda, K. 2011. Development of PCR markers for Tamyb10 related to R-1, red grain color gene in wheat. Theor. Appl. Genet. 122:1561-1576.

Himi, E., Taketa, S. 2015. Isolation of candidate genes for the barley Ant1 and wheat Rc genes controlling anthocyanin pigmentation in different vegetative tissues. Mol. Genet. Genomics 290:1287-1298.

Huang, Y.J., Song, S., Allan, A.C., Liu, X.F., Yin, X.R., Xu, C.J., Chen, K.S. 2013. Differential activation of anthocyanin biosynthesis in Arabidopsis and tobacco over-expressing an R2R3 MYB from Chinese bayberry. Plant Cell Tiss. Org. Cult. 113:491-499.

Joppa, L.R., Williams, N.D. 1988. Langdon durum disomic substitution lines and aneuploid analysis in tetraploid wheat. Genome 30:222-228.

Khlestkina, E.K. 2013. Genes determining the coloration of different organs in wheat. Russ. J. Genet. Appl. Res. 3:54-65.

Khlestkina, E.K., Pestsova, E.G., Röder, M.S., Börner, A. 2002. Molecular mapping, phenotypic expression and geographical distribution of genes determining anthocyanin pigmentation of coleoptiles in wheat (Triticum aestivum L.). Theor. Appl. Genet. 104:632-637.

Khlestkina, E.K., Röder, M.S., Börner, A. 2010. Mapping genes controlling anthocyanin pigmentation on the glume and pericarp in tetraploid wheat (Triticum durum L.). Euphytica 171:65-69.

Kobayashi, S., Yamamoto, N.G., Hirochika, H. 2005. Association of VvmybA1 gene expression with anthocyanin production in grape (Vitis vinifera) skin - color mutants. J. Jap. Soc. Hort. Sci. 74:196-203.

Koes, R., Verweij, W., Quattrocchio, F. 2005. Flavonoids: a colorful model for the regulation and evolution of biochemical pathways. Trends Plant Sci. 10:236-242.

Liu, D., Li, S., Chen, W., Zhang, B., Liu, D., Liu, B., Zhang, H. 2016. Transcriptome analysis of purple pericarps in common wheat (Triticum aestivum L.). PLoS One 11: e0155428.

Mano, H., Ogasawara, F., Sato, K., Higo, H., Minobe, Y. 2007. Isolation of a regulatory gene of anthocyanin biosynthesis in tuberous roots of purple-fleshed sweet potato. Plant Physiol. 143:1252-1268.

Peel, G.J., Pang, Y.Z., Modolo, L.V., Dixon, R.A. 2009. The LAP1 MYB transcription factor orchestrates anthocyanidin biosynthesis and glycosylation in Medicago. Plant J. 59:136-149.

Quattrocchio, F., Wing, J., van der Woude, K., Souer, E., de Vetten, N., Mol, J., Koes, R. 1999. Molecular analysis of the anthocyanin2 gene of petunia and its role in the evolution of flower color. Plant Cell 11:1433-1444.

Saitoh, K., Onishi, K., Mikami, I., Thidar, K., Sano, Y. 2004. Allelic diversification at the $C(O s C 1)$ locus of wild and cultivated rice. Genetics 168:997-1007.

Sheen, J. 2002. A transient expression assay using Arabidopsis mesophyll protoplasts. Dept. Mol. Biol., Mass. Gen. Hosp., Boston, MA. http://molbio.mgh.harvard.edu/sheenweb/protocols/AtprotoRL04.pdf 
Shoeva, O.Y., Gordeeva, E.I., Khlestkina, E.K. 2014. The regulation of anthocyanin synthesis in the wheat pericarp. Molecules 19:20266-20279.

Tamura, K., Dudley, J., Nei, M., Kumar, S. 2007. MEGA4: Molecular evolutionary genetics analysis (MEGA) software version 4.0. Mol. Biol. Evol. 24:1596-1599.

Tereshchenko, O.Y., Gordeeva, E.I., Arbuzova, V.S., Börner, A., Khlestkina, E.K. 2012. The D genome carries a gene determining purple grain colour in wheat. Cereal Res. Commun. 40:334-341.

Wang, H.W., Wang, Z.L., Wang, P., Wang, S.G., Huang, W., Wu, Y.G., Sun, L.Z., Yin, Y.P. 2011. Effect of shading post anthesis on anthocyanin accumulation and activities of related enzymes in colored-grain wheat. Acta Agron. Sin. 37:1093-1100 (in Chinese with English abstract).

Wang, Y.Q., Hou, X.J., Zhang, B., Chen, W.J., Liu, D.C., Liu, B.L., Zhang, H.G. 2016. Identification of a candidate gene for $R c-D 1$, a locus controlling red coleoptile colour in wheat. Cereal Res. Commun. 44:35-46.

Wei, Y.L., Li, J.N., Lu, J., Tang, Z.L., Pu, D.C., Chai, Y.R. 2007. Molecular cloning of Brassica napus TRANSPARENT TESTA 2 gene family encoding potential MYB regulatory proteins of proanthocyanidin biosynthesis. Mol. Biol. Rep. 34:105-120.

Xu, W., Dubos, C., Lepiniec, L. 2015. Transcriptional control of flavonoid biosynthesis by MYB-bHLH-WDR complexes. Trends Plant Sci. 20:176-185.

Yoo, S.D., Cho, Y.H., Sheen, J. 2007. Arabidopsis mesophyll protoplasts: A versatile cell system for transient gene expression analysis. Nat. Protoc. 2:1565-1575.

Zeven, A.C. 1991. Wheats with purple and blue grains: a review. Euphytica 56:243-258.

\section{Electronic Supplementary Material (ESM)}

Electronic Supplementary Material (ESM) associated with this article can be found at the website of CRC at http://www.akademiai.com/content/120427/

Electronic Supplementary Table S1. Names and sequences of the primers used in this study

Electronic Supplementary Figure S1. Conserve primers design and amplification with cDNA of Gy115 grain $7 \mathrm{~d}$ after blossom. A, TaC1-F and TaC1-R were designed in the first and third extrons close to the introns. The arrows show the sites of the primers. (OsC1, KJ934847.1; AtTT2, NC 003076; ZmC1, AF320613), B, 7(L) represents the amplification product from cDNA of Gy115 $7 \mathrm{~d}$ after blossom with dark treatment (Land 1), and 7(d) is the dark treatment. The objective land was 220 bp (Land 2). Land 3 was the marker

Electronic Supplementary Figure S2. The TaMYB3 location on chromosome 4BL 